\title{
Posible efecto neuroprotectivo del sulfato de magnesio en recién nacidos pretérmino
}

\section{Objetivo}

Determinar la efectividad neuroprotectiva del sulfato de magnesio $\left(\mathrm{MgSO}_{4}\right)$ administrado a mujeres en riesgo de parto pretérmino (antes de las 30 semanas) para disminuir en el recién nacido el riesgo de muerte y el desarrollo de parálisis cerebral.

\section{Diseño}

Ensayo controlado aleatorizado con seguimiento de los niños sobrevivientes hasta los dos años de edad corregida.

\section{Lugar}

Dieciséis hospitales terciarios en Australia y Nueva Zelanda, estratificados por centro y por embarazo múltiple.

\section{Pacientes}

Se enrolaron 1062 mujeres (535 asignadas a recibir $\mathrm{MgSO}_{4}$ y 527 a placebo) con fetos menores a 30 semanas de gestación, cuyo nacimiento fuera planeado o esperado en las siguientes $24 \mathrm{~h}$.

\section{Intervención}

Las mujeres fueron asignadas aleatoriamente a recibir una infusión de $8 \mathrm{~mL}\left(4 \mathrm{~g}[16 \mathrm{mmol}]\right.$ de $\mathrm{MgSO}_{4}$ en el grupo intervención o solución de cloruro de sodio isotónico [0,9\%] en el grupo placebo) durante 20 minutos, seguido por una infusión de mantenimiento de $2 \mathrm{~mL} / \mathrm{h}$ hasta el nacimiento ó durante $24 \mathrm{~h}$. El sulfato de magnesio fue dado solamente como agente neuroprotector.

\section{Medición de resultados principales}

Los resultados principales a evaluar fueron tres: 1) tasa de mortalidad pediátrica; 2) tasa de parálisis cerebral; 3) resultado combinado* de muerte o parálisis cerebral (PC) a la edad corregida de 2 años.

\section{Resultados principales}

Los datos fueron analizados en 1047 (99\%) sobrevivientes a los dos años años de edad corregida y los principales resultados se describen en la tabla. Los tres resultados principales definidos en el diseño del estudio (mortalidad pediátrica total, riesgo de PC en sobrevivientes y resultado combinado "muerte o PC") fueron menos frecuentes en infantes expuestos a $\mathrm{MgSO}_{4}$, pero ninguna de estas diferencias fue estadísticamente significativa. Sin embargo, la disfunción motora grosera y el resultado combinado "muerte o disfunción motora grosera" sí mostró diferencias estadísticamente significativas a favor del grupo tratado con $\mathrm{MgSO}_{4}$.

Tabla: Resultados principales

\begin{tabular}{|c|c|c|c|}
\hline $\begin{array}{l}\text { Resultado en el recién nacido } \\
\text { (proporción de pacientes) }\end{array}$ & $\begin{array}{l}\mathrm{MgSO}_{4} \\
(\mathrm{n}=535)\end{array}$ & $\begin{array}{l}\text { Placebo } \\
(n=527)\end{array}$ & $\begin{array}{l}\text { Riesgo Relativo } \\
\text { (IC95\%) }\end{array}$ \\
\hline Muerte & $13,8 \%$ & $17,1 \%$ & $0,83(0,64$ a 1,09$)$ \\
\hline Parálisis cerebral en sobreviviente & $6,8 \%$ & $8,2 \%$ & $0,83(0,54$ a 1,27$)$ \\
\hline Parálisis cerebral o muerte & $19,8 \%$ & $24 \%$ & $0,83(0,66$ a 1,03 \\
\hline Disfunción motora grosera ("sustancial") & $3,4 \%$ & $6,6 \%$ & $0,51(0,29$ a 0,91$)$ \\
\hline $\begin{array}{l}\text { Disfunción motora grosera ("sustancial") } \\
\text { o muerte }\end{array}$ & $17 \%$ & $22,7 \%$ & $0,75(0,59$ a 0,96$)$ \\
\hline
\end{tabular}

\section{Conclusiones}

La administración antenatal en mujeres de $\mathrm{MgSO}_{4}$ inmediatamente antes de dar a luz a niños menores a 30 semanas de edad gestacional podría mejorar los resultados clínicos pediátricos. No se hallaron diferencias significativas en los resultados de mortalidad total, PC y combinado de mortalidad o PC aunque todos ellos fueron menores en el grupo magnesio. Se observó una reducción estadísticamente significativa en la disfunción motora sustantiva entre los sobrevivientes en el grupo $\mathrm{MgSO}_{4}$ y en el combinado mortalidad o disfunción motora sustancial, ambas consideradas clínicamente importantes.

Fuente de financiamiento: National Health and Medical Research Council Australia,Research Foundation of South Australia Inc, and the Queen Victoria Hospital Research Foundation, Adelaide, South Australia.

\section{Comentario}

El promedio de $\mathrm{MgSO}_{4}$ antenatal utilizado en este estudio para neuroprotección fue de 10,5g/dosis. En dos ensayos clínicos controlados realizados previamente, Mittendorf ${ }^{1}$ y $\mathrm{Cox}^{2}$ encontraron que dosis de tocolisis (50g ó más) aumentaban el riesgo de mortali-dad total en los niños expuestos.

Los beneficios observados en el presente estudio fueron menores que los esperados a partir de los resultados de estudios previos no aleatorizados, mientras que los riesgos de mortalidad y parálisis cerebral (resultados primarios) fueron menores que los originalmente predichas. Analizados los resultados secundarios, tampoco se hallaron diferencias significativas en la incidencia de hemorragia intraventricular (HIV) leucomalacia periventricular (LPV) enfermedad pulmonar crónica (EPC) ni enterocolitis necrotizante (ECN) o bien en la duración de la ventilación mecánica entre los niños expuestos y los no expuestos. Tampoco hubo diferencias significativas cuando los grupos fueron comparados respecto de sus discapacidades neurosensoriales o bien respecto de la presencia y severidad de alteraciones neurosensoriales específicas (parálisis cerebral, ceguera, sordera o retraso en el desarrollo). Pero cuando se usó la Clasificación Motora Gruesa desarrollada por Palisano y $\mathrm{Col}^{3}$, se observó en los niños prematuros (menos de 30 semanas de EG) expuestos a sulfato de magnesio una importante reducción en la incidencia de la disfunción motora sustancial a los dos años de edad corregida (reducción de riesgo relativo* 48\%). El beneficio atribuible* a la intervención para la prevención de la disfunción motora sustancial fue $3,2 \%$. En cuanto al resultado combinado de muerte o disfunción motora sustancial, los autores hallaron una disminución de $25 \%$ en la incidencia de una u otra en los niños expuestos, con un beneficio atribuible a la intervención de 5,7\%. Según el estudio, el número necesario a tratar para reducir un caso de discapacidad motora sustancial es 32 niños y para evitar un caso de muerte o discapacidad severa, 18 niños. De los 52 niños con disfunción motora grosera, 39 tenían PC (3 mínima, 27 moderada y 9 severa) pero el trabajo no aclara cuántos de estos niños con PC pertenecían a cada grupo. Una limitación del estudio fue que no hubo expertos entrenados para el diagnóstico de PC ya que la misma fue diagnosticada como reflejo de la práctica clínica habitual.

\section{Conclusiones del comentador}

Tal como los autores señalan, los beneficios hallados necesitan ser confirmados con futuras investigaciones y el uso de sulfato de magnesio como agente neuroprotector no debe ser recomendado solamente en base a este estudio.

Elsa Andina [ División Obstetricia. Maternidad Sardá.]

Andina E. Posible efecto neuroprotectivo del sulfato de magnesio en recién nacidos pretérmino. Evid actual pract ambul 2005;8:37. Comentado de: Crowther C, Hiller J, Doyle L. Effect of magnesium sulfate given for neuroprotection before preterm birth: a randomized controlled trial. JAMA. 2003 Nov 26;290(20):2669-76. PMID: 14645308.

\section{Referencias}

1. Mittendorf R, Covert R, et al. Is tocolytic magnesium sulphate associated with increased total paediatric mortality? Lancet.1997;350;1517-1518

2. Cox SM, Sherman ML, Leveno KJ. Randomized investigation of magnesium sulfate for prevention of preterm birth. Am J Obstet Gynecol. 1990;163:767-772

3. Palisano R, Rosenbaum P, Walter S, Russell D, Wood E, Galuppi B. Development and reliability or a system to classify gross motor function in children whith cerebral palsy. Dev Med Child Neurol 1997,39:214-223 